\title{
Variations in Hardness with Position In One Dimensionally Recovered Shock Loaded Metals
}

\author{
G. Whiteman ${ }^{1, *}$, D.L. Higgins ${ }^{2}$, B. Pang ${ }^{2}$, J.C.F. Millett ${ }^{1}$, Y-L. Chiu ${ }^{2}$ and I.P. Jones ${ }^{2}$ \\ ${ }^{1}$ AWE plc, Aldermaston, Reading, RG7 4PR. United Kingdom \\ ${ }^{2}$ School of Metallurgy and Materials, University of Birmingham, Elms Road, Edgbaston, Birmingham, B15 2TT. United \\ Kingdom
}

\begin{abstract}
The microstructural and mechanical response of materials to shock loading is of the utmost importance in the development of constitutive models for high strain-rate applications. However, unlike a purely mechanical response, to ensure that the microstructure has been generated under conditions of pure one dimensional strain, the target assembly requires both a complex array of momentum traps to prevent lateral releases entering the specimen location from the edges and spall plates to prevent tensile interactions (spall) affecting the microstructure. In this paper, we examine these effects by performing microhardness profiles of shock loaded copper and tantalum samples. In general, variations in hardness both parallel and perpendicular to the shock direction were small indicating successful momentum trapping. Variations in hardness at different locations relative to the impact face are discussed in terms of the initial degree of cold work and the ability to generate and move dislocations in the samples.
\end{abstract}

\section{Introduction}

To successfully predict the physical behaviour of materials subjected to dynamic loads requires an understanding of, and ability to simulate, the underlying microscopic processes that govern the macroscopic response. Under ideal circumstances it would be desirable to obtain a snapshot of the microstructural configuration inside a sample during shock loading. In practice, while some microstructural aspects can be measured using x-ray diffraction techniques [1], such experiments are generally limited to thin targets (micrometres) during short duration (nanoseconds) laser loading experiments.

Shock loading over a period of a few microseconds, over millimetre distances, relevant to many real world applications, requires plate-impact or explosive loading techniques. The viable option for studying microstructural response for these types of test is the post-shock metallographic study of samples, i.e. those which have been through a shock sequence consisting of a loading pulse, potentially a period held at pressure and finally a release back to ambient conditions. Each of these stages adds its own contributions to the deformation such that the microstructure obtained is cumulative in nature for the full loading/unloading event. To pin-point individual deformation mechanisms caused by the uniaxial strain event also requires the removal of the effects of radial release waves and tension effects in the sample. A technique to achieve this was first developed by Smith [2] for explosively loaded samples and was later modified by Gray and colleagues [3-7] for use on single stage gas-guns. This work has been adapted by the current authors to study polycrystalline copper [8-10] and tantalum [9, 10].

One-dimensional shock recovery experiments generally consist of a number of closely fitting rings and plates surrounding a plate of the specimen material. The manufacturing tolerances for the momentum trapping rings have been shown by Gray and colleagues [11] to be of primary importance to the reduction of radial release wave effects. Interference fits between a sample and inner momentum trapping ring and slip fit tolerances of $(25.4 \mu \mathrm{m})$ between additional rings were shown to reduce residual plastic strains to $<2 \%$. However, even with excellent manufacturing, mating surfaces can never be completely seamless such that additional small wave effects will always be present, which can affect local material deformation. In most cases the product of shock recovery research is deductions regarding active microstructural deformation mechanisms determined from the microscale study of the material structure (e.g. from transmission electron microscopy (TEM)) or the macroscale study of the material response (e.g. using mechanical compression tests). Studies of hardness can be used to bridge these scales, offering quantitative assessment of material response on the microscale but undertaken across wide regions of the sample.

In this paper we use microhardness measurements of shock-recovered samples to help understand the positional variations induced, using this to assess the efficacy of the experiments. 


\section{Experimental}

All shots were fired on a $70 \mathrm{~mm}$ bore, single stage gasgun. Samples of $\sim 30 \mathrm{~mm}$ diameter and $5 \mathrm{~mm}$ thick, pure polycrystalline copper and tantalum were mounted in target assemblies matched to the sample material, similar to the example shown in figure 1. Impact stresses in the range $5.1-9.6 \mathrm{GPa}$ (copper) and 7.0 - 24.2 GPa (tantalum) were induced using $65 \mathrm{~mm}$ diameter impactors material-matched to the samples, impacting targets at velocities in the range $\sim 230-750 \mathrm{~m} / \mathrm{s}$. Impactor thicknesses (approximately $2 \mathrm{~mm}$ ) were chosen to produce square pulse loading times of $1 \mu \mathrm{s}$. As shown in the figure a series of rings and plates surrounded the sample in each case to minimise the effects of lateral momentum and tension entering the sample. To aid soft recovery in both series the inner momentum trapping rings were manufactured following the methods of Gray [7] from slightly stronger materials $(\mathrm{Ta} 2.5 \% \mathrm{~W}$ and phosphor bronze respectively) which have almost identical shock impedance to the samples.

a)

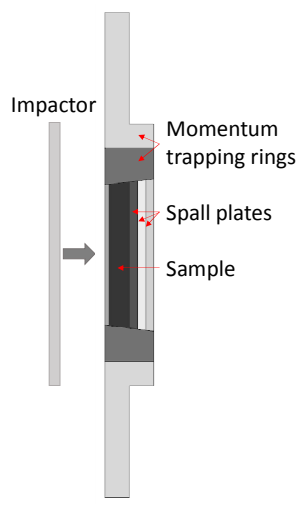

c)

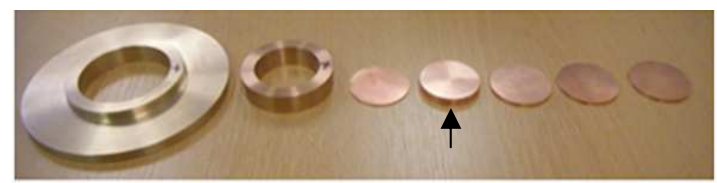

Fig. 1. Typical target assembly for a plate impact recovery experiment: a) sketch of sectioned assembly, b) exploded view and c) disassembled target. The specimen is highlighted by the arrow.

The machining tolerances in these experiments ensured that the flatness of all surfaces parallel to the impact plane and the mating of all radial interfaces were better than $5 \mu \mathrm{m}$. These target designs ensured that the samples were loaded and unloaded under conditions of purely one dimensional strain. In order to subsequently bring samples to rest whilst preventing further damage, they were decelerated using bags containing a hydrated mixture of sodium polyacrylate and wood pulp. This also quenched the samples back to ambient temperature.

\section{Materials}

The sample materials used in these experiments were pure polycrystalline tantalum $(99.98 \%$ ) and oxygen free high conductivity copper $(99.99 \%)$. The tantalum was obtained as $75 \mathrm{~mm}$ diameter, $1 \mathrm{~m}$ long bar stock with a mean grain size of $\sim 120 \pm 20 \mu \mathrm{m}$ in the inner $50 \mathrm{~mm}$ diameter region. Some banding of grain sizes was present in the microstructure, particularly notable near the bar edge with much larger grains present. Asreceived samples were cut from the central portion of the bar. The material displayed a preferred grain orientation along the loading axis in the $<001>$ direction. Additionally some of the bar stock material was taken and cold rolled perpendicular to the bar axis to $50 \%$ reduction in thickness. Samples were also used from this material although they were generally taken from locations equivalent to nearer the original bar stock edge and therefore had a larger range of grain sizes present. Transmission electron micrographs showed that in the as-received state, dislocation density was relatively low, with the dislocations arranged in a loose cell structure. In the cold-rolled material, dislocation density appeared to have increased by only a small amount, although they were arranged into a more clearly defined cell structure. The Vickers hardness of the original materials was measured to be $89.5 \mathrm{HV}$ and $135.1 \mathrm{HV}$ for the annealed and rolled conditions respectively. Further details of the tantalum are given elsewhere [12].

The copper was bought as $25 \mathrm{~mm}$ plate stock in the half hard condition, corresponding to an approximate reduction in thickness of $21 \%$. An equiaxed grain size of ca. $180 \mu \mathrm{m}$, including annealing twins, was measured using EBSD. To generate a low dislocation microstructure some of the material was annealed at 450 ${ }^{\circ} \mathrm{C}$ for 1 hour, followed by an air cool. The annealed material has a low dislocation density of ca. $3.8 \times 10^{8}$ $\mathrm{cm}^{-2}$, and a grain size of ca. $64 \mu \mathrm{m}$. In contrast, the half hard material has a much higher dislocation density arranged in a cell network, although it has not been quantified. The Vickers hardness of the original materials was measured to be $50.8 \mathrm{HV}$ and $91.0 \mathrm{HV}$ for the annealed and half hard conditions respectively.

\section{Results}

All samples were successfully recovered with minimal indications of deformation or residual strain indicating effective momentum trapping. Measured longitudinal engineering strains were $<1 \%$ for most samples and $\sim 1.5 \%$ for the cold-rolled tantalum sample shocked to $24.2 \mathrm{GPa}$. Recovered samples were investigated via electron microscopy techniques, post-shock compression tests and microhardness measurements which are reported here. Microhardness profiles were measured using a peak force of $0.2 \mathrm{~N}$ on a Struers DuraScan at impact and rear surfaces and through the thicknesses of all samples. The through thickness measurements, taken at intervals of $100 \mu \mathrm{m}$, are shown in figure 2. Each data point shown is the mean of 10 measurements. Standard deviations of the data points were consistently $1-5 \mathrm{HV}$ for the copper samples and 3-10 HV for tantalum.

Figure 2a shows the hardness profiles for the recovered copper materials compared to the unshocked source material. The data shows the expected overall increase in hardness caused by the shock loading of 
$\sim 48 \%$ and $5 \%$ for the annealed and cold rolled materials respectively at the lowest impact stresses and $14 \%$ for the cold-rolled copper shocked to $9.6 \mathrm{GPa}$. In all cases the through thickness hardness values are approximately constant, within error, with a small upturn in the last $\sim 2 \mathrm{~mm}$.

Figure $2 \mathrm{~b}$ shows the hardness data for tantalum. As with copper the overall hardness increases with shock pressure for both annealed and cold rolled materials. In general the shock-recovered cold rolled material is slightly harder than the annealed material although after a $24.2 \mathrm{GPa}$ shock both materials respond in a very similar manner. Compared to the unshocked source materials the as-received material has increased its hardness significantly (by $\sim 35 \%$ at a shock stress of 7.0 $\mathrm{GPa}$ ) whereas the hardness of the cold-rolled material at the same shock stress is actually a few percent less than that of the original material. The cold rolled tantalum recovered after a $15.6 \mathrm{GPa}$ shock is clearly behaving differently to the other materials. While for the other samples there is a general trend of a small reduction in hardness with distance from the impact face, the hardness of the $15.6 \mathrm{GPa}$ cold-rolled sample increases with distance across the whole sample. There is also a more significant upturn at $\sim 2 \mathrm{~mm}$ from the rear surface.

a)

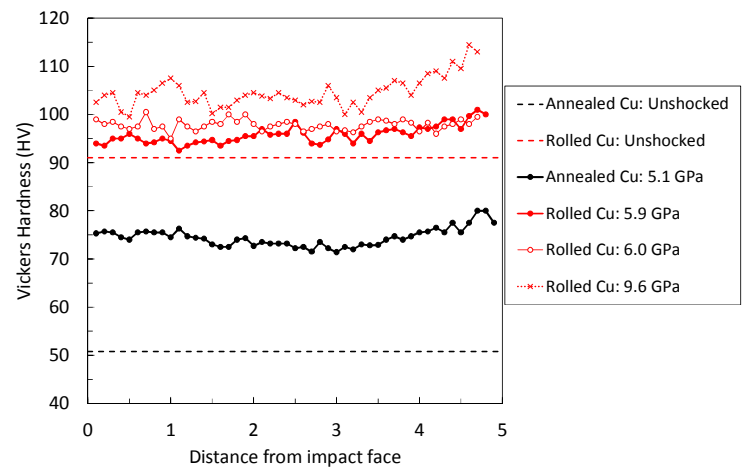

b)

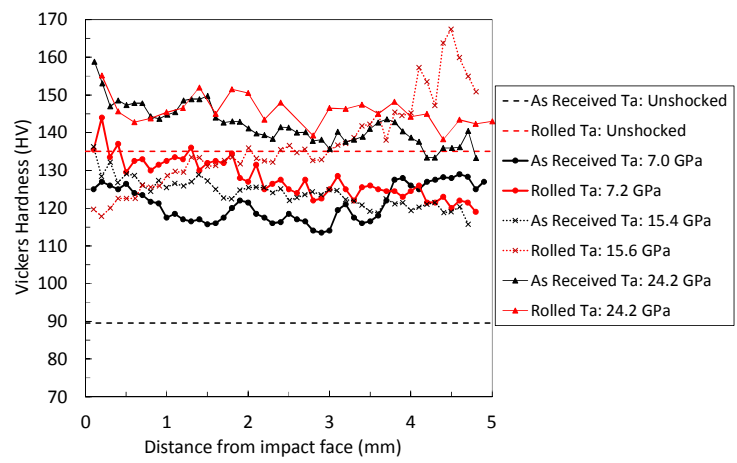

Fig. 2. Vickers hardness vs distance from the impact surface of shock-recovered samples for a) annealed and cold-rolled copper and b) as-received and cold-rolled tantalum.

In order to check the efficacy of the momentum trapping rings in the recovery assembly, a series of hardness measurements were also undertaken on the sample surfaces perpendicular to the shock direction. In figure 3 examples of these are shown for the front surface of the cold rolled copper shocked to $6 \mathrm{GPa}$ and the as-received and cold rolled tantalum shocked to $\sim 7$ $\mathrm{GPa}$. a)

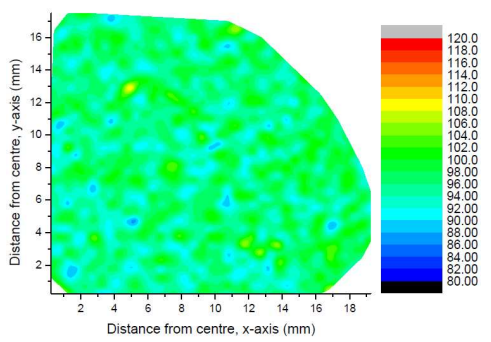

b)

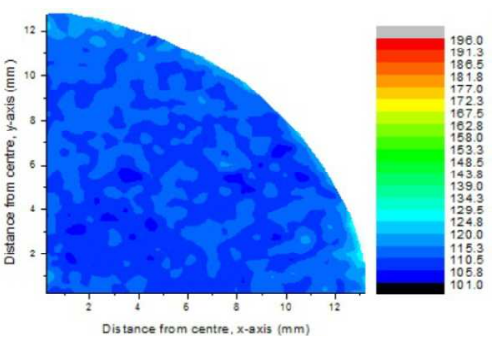

c)

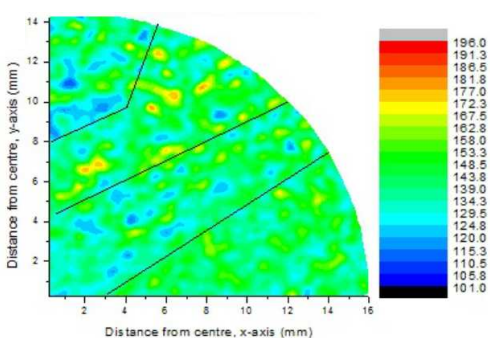

Fig. 3. Hardness contour maps from the front surface of the shock recovered samples; a) cold rolled copper shocked and recovered at $6.0 \mathrm{GPa}, \mathrm{b}$ ) as-received tantalum shocked and recovered at $7.0 \mathrm{GPa}$ c) cold-rolled tantalum shocked and recovered at $7.2 \mathrm{GPa}$.

No significant variations were observed at the sample edges as seen in figure 3, indicating successful lateral momentum trapping. The typical example for copper in figure $3 \mathrm{a}$ shows that there was only minimal variation observed perpendicular to the shock axis. This confirms that the variation with thickness, such as the last $2 \mathrm{~mm}$ upturn shown in figure $2 \mathrm{a}$, is consistent throughout the samples.

In figures $3 b$ and $3 c$ the as-received and cold rolled tantalum samples are shown using the same colour scale. The as-received tantalum samples displayed minimal radial variation, similar to copper. For the cold-rolled material however, variations in the form of local spots of high and low hardness are observed which appear in approximate bands, highlighted using the black lines in figure $3 \mathrm{c}$. This was confirmed using electron back scatter diffraction images to be due to grain size variation, present from the original bar stock. Whereas the asreceived samples, taken from near the bar centre had grain-sizes in the range $\sim 106-128 \mu \mathrm{m}$, the rolled samples, effectively taken from nearer the bar edge, had a much larger grain size variation with some very large grains of almost $1000 \mu \mathrm{m}$. It is expected that this grain size variation which was probably columnar in the original material, once rolled will effectively have also created variation through thickness like that seen in figure $2 \mathrm{~b}$ for the $15.6 \mathrm{GPa}$ shocked rolled tantalum sample. 


\section{Discussion}

The purpose of recovery experiments is to provide specimens that have be loaded and released back to ambient under conditions of purely one dimensional strain, without additional complications from spall interactions. Out of necessity, the target assembly has to include a number of interfaces that ideally prevent the transmission of releases from the edges of the sample (lateral) and rear surface into the specimen itself. Under perfect conditions, the whole target would then behave as a monolithic assembly during the loading phase, the various interfaces only reacting during release. Unfortunately, it is not possible to make a perfect interface, and thus there exists the potential for the presence of these interfaces in the target assembly to have an adverse effect upon the recovered microstructure.

Probably the area of greatest concern is the lateral momentum traps. Failure to prevent lateral releases entering the sample would result in three dimensional loading. The hardness profile maps presented in figure 3 go a long way to alleviating these concerns. In the case of cold rolled copper and as-received tantalum (shocked to 6.0 and $7.0 \mathrm{GPa}$ respectively), very little variation in hardness can be seen over the entire area. Note in particular that there is no evidence of hardness deviations around the outer edges of these samples, which would be indicative of inadequate release capture across those interfaces. In the case of the cold rolled tantalum shocked to $7.2 \mathrm{GPa}$, also note that there is no evidence of hardness changes around the outer edge, again indicating successful release capture. However, it should be pointed out that the hardness mapping does indicate a banded structure, as indicated by the solid lines in figure $3 \mathrm{c}$. We believe that this is a manifestation of the variable microstructure of the tantalum in its asreceived state. Higgins [9] showed that the grain size varied from $60 \mu \mathrm{m}$ to $1 \mathrm{~mm}$ depending on location. It is thus likely that the variations in harness seen in figure $3 \mathrm{c}$ are due to this.

Variations of hardness along the loading axis are presented in figure 2. In the case of copper (figure 2a), shock loading annealed copper to $5.1 \mathrm{GPa}$ causes an increase in hardness of $c a .48 \%$, whilst shock loading the cold rolled material to stresses of 6 and $9.8 \mathrm{GPa}$ increases hardness by $c a .5$ and $14 \%$ respectively. This clearly shows that the annealed material under shock loading experiences a huge amount of dislocation generation, despite the comparatively low levels of strain, due to the dislocation velocity being too low to accommodate deformation without further generation. This is consistent with the microstructural observations from both ourselves $[9,10]$ other workers [13]. Note that cold rolled material still possesses the ability to generate additional dislocations during shock loading, although at a lower level, again consistent with previous microstructural and mechanical observations $[9,10]$. The situation with tantalum is more complex. In the case of the as received material, shock loading results in an increase of hardness of ca. $35 \%$ at $7 \mathrm{GPa}$ and $55 \%$ at $24.2 \mathrm{GPa}$. These results are superficially similar to annealed copper and suggest that significant dislocation generation is occurring during shock loading that increases with shock pressure. In the case of cold rolled tantalum, material shocked to $7.2 \mathrm{GPa}$ appears softer than in its original unshocked state, whilst the material shocked to $24 \mathrm{GPa}$ displays minimal hardening only with respect to the unshocked state. We believe that this is due to the role of interstitial solute atoms (principally oxygen). In a previous work [12], we showed that after a prior cold rolling treatment, the HEL actually reduced with respect to its annealed counterpart. This was explained in terms of oxygen atoms preferentially locating around dislocations (due to local lattice distortions) which effectively pins the dislocations in place. The effect of prior cold rolling is to move the dislocations whilst leaving the oxygen atoms in place. Therefore as long as dislocation build up during cold rolling is not too great, it can be seen that strength (either during shock loading itself or post shock hardness measurements) can be reduced, even to the point where strength is lower than in the initial unshocked state. Note that the rolled specimen shocked to $15.6 \mathrm{GPa}$ shows anomalous behaviour. We believe that this is a manifestation of the variable grain size in the rolled tantalum sample, as highlighted by the banding in figure 3c.

The final point to discuss is the variation of hardness with position through the sample. In the case of copper (figure 2a), it can be seen that at approximately $3 \mathrm{~mm}$ from the impact face, there is a small increase in hardness that increases towards the rear surface of the specimen, behind which lie the first of the plates designed to capture any spallation response. As stated previously, under ideal circumstances, these spall plates would allow passage of the shock front without interference, and thus any spallation from interactions between releases from the flyer plate and target assembly should occur within the spall plates. However, it is impossible to manufacture a perfect interface, and hence in reality a gap of the order of a few microns exists. As a consequence it is possible that a partial release from the rear of the sample could travel back to interact with the release from the flyer plate. For example, a copper sample shocked to $5.87 \mathrm{GPa}$ will have a shock velocity

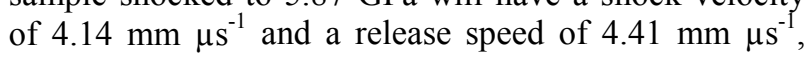
resulting in the releases meeting $2.9 \mathrm{~mm}$ from the rear surface of the specimen. The situation with the tantalum hardness profiles (figure $2 \mathrm{~b}$ ), with the exception of the cold rolled sample shocked to $156 \mathrm{GPa}$ (discussed above), seems relatively consistent, with a gradual decline in hardness from the impact face. Note that in the annealed material shocked to $7 \mathrm{GPa}$, there is an upturn at $3 \mathrm{~mm}$, similar to the results from copper, and most likely for the same reasons. The gradual decline in hardness is likely due to the gradual decrease in pulse width due to the higher release speed in comparison to the shock velocity. Another possibility lies in the differences in dislocation mobility between copper and tantalum. Note that until the upturn at $3 \mathrm{~mm}$, the hardness with distance from impact in copper is near constant, whilst in tantalum, the general trend is to decrease. In common with other face centred cubic metals, the Peierls stress is 
very low, thus allowing rapid motion and generation. This manifests as an extremely fast rise in the plastic part of the shock front. The elastic part of the shock front will travel faster than the plastic, resulting in an increase in rise time from the elastic limit to the final shock stress. In turn this will reduce the strain-rate in the plastic front, from which one would expect a reduction in dislocation activity and a corresponding reduction in hardness from the impact face. That these effects are not observed in copper is most likely due to the extremely fast plastic rise reducing only slightly, and thus any reduction in hardness is probably masked by the statistical variation due to microstructure. The situation with tantalum is different. In this case, the much higher Peierls stress results in much reduced dislocation mobility and generation. Therefore, it takes longer for tantalum to achieve the required dislocation density for the applied deformation, hence a longer plastic rise time. Further the plastic rise will slow significantly as it travels through the specimen, with a corresponding reduction in strainrate and hence reduction in hardness.

\section{Conclusions}

A series of one dimensional strain, shock recovery experiments on tantalum and copper in annealed and cold worked states have been reported. Microhardness measurements taken over the area of the recovered sample showed no variations that could be attributed to releases originating from the lateral edges of either that target assembly or the specimen itself. Therefore we can be confident that the samples were loaded, released and recovered purely under conditions of one dimensional strain. Hardness profiles through the thickness of the samples showed, in a majority of experiments, that there was an effect. In the case of copper, an increase in hardness approximately $3 \mathrm{~mm}$ from impact correlated with the position of interaction between releases from the rear of the flyer and a partial release from the rear of the specimen plate, indicating that the interfaces between the rear of the specimen and the first spall plate were not perfect, thus allowing a partial release to travel back into the target. In the case of tantalum, the majority of hardness profiles showed a gradual decline with distance from the impact surface. We have suggested that this is a combination of reducing pulse width due to the higher release velocity (in comparison with the ingoing shock velocity) and increasing dispersion between the elastic and plastic wave fronts reducing strain-rate as the shock front passes through the specimen. However, we point out that these changes of hardness are small (and in some cases it could be argued that they are within the statistical variation of the measurements anyway), and thus their effects on the ultimate microstructure are minimal. We are therefore confident that the design of these sets of experiments was sufficient to provide valid post-shock microstructural and mechanical data on both copper and tantalum. Further, we advocate the use of hardness profiles as a quantitative assessment of microscale mechanical response which can be performed across a macroscale sample. As such, studies of hardness can be used to bridge the microscale observations of electron microscopy and the macroscale mechanical response of compression testing whilst providing a useful diagnostic to check that shock recovered specimens have only been loaded and released back to ambient conditions under one dimensional strain.

C British Crown Owned Copyright 2018/AWE.

\section{References}

1. D. McGonegle, A. Higginbotham, E. Galtier, E.E. McBride, M.I. McMahon, D. Milathianaki, et al. J Phys: Conf Series;500:112063. (2014)

2. C.S. Smith. Trans Met Soc AIME;212:574-85. (1958)

3. N.K. Bourne, G.T. Gray III. Proc R Soc A;461:3297-312. (2005)

4. G.T. Gray III, editor Shock Recovery Experiments: An Assessment. Shock Compression of Condensed Matter 1989: North-Holland: Amsterdam (1990)

5. G.T. Gray III. Deformation substructures induced by high rate deformation, in Modelling the deformation of crystalline solids, . Lowe TC, Rollett AD, Follansbee PS, Daehn GS, editors, pp 145-58 ( 1991)

6. G.T. Gray III. Shock experiments in metals and ceramics, in Shock-wave and high-strain-rate phenomena in materials. Meyers MA, Murr LE, Staudhammer KP, editors. New York: Marcel Dekker (1992).

7. G.T. Gray III. Influence of shock-wave deformation on the structure/property behaviour of materials. Asay JR, Shahinpoor M, editors. New York: Springer-Verlag (1993)

8. D.L. Higgins, B. Pang, J.C.F. Millett, G. Whiteman, I.P. Jones, Y.-L. Chiu. Met Mat Trans A;45A:4518-21 (2014)

9. D.L. Higgins. High Strain Rate Deformation of Copper, Titanium 6Al-4V and Tantalum and QuasiStatic Compression of Tantalum. Birmingham: University of Birmingham (2017)

10. J.C.F. Millett, D.L. Higgins, G. Whiteman, B. Pang, Y.-L. Chiu, I.P. Jones, editors. Contrasting the Effects of Cold Rolling on the Shock Response of Typical Face Centred Cubic and Body Centred Cubic Metals. Proceedings of the American Physical Society Topical Group Meeting on Shock Compression of Condensed Matter; 2017: (in press).

11. G.T. Gray III, P.S. Follansbee, C.E. Frantz. Mater Sci Engng;A111:9-16 (1989)

12. J.C.F. Millett, G. Whiteman, N.T. Park, S. Case, N.K. Bourne. J App Phys;113:233502 (2013)

13. G.T. Gray III, C.E. Morris. Journal de Physique IV, Colloque C3, suppl au Journal de Physique III;1:1916 (1991) 
